\title{
Using Response Latency within a Preference Assessment
}

\author{
Stephanie K. Meador, K. Mark Derby, T. F. McLaughlin, \\ Anjali Barretto and Kim Weber
}

This study evaluated the effects of using differential reinforcement of other behavior (DRO) with a differential reinforcement of alternative behavior (DRA) resetting time schedule to reduce stereotypy in a child with Rett Syndrome. The primary purpose of the investigation was to compare latency and choice as dependent measures to identify reinforcers for use within a DRO/A contingency using a resetting time schedule. The treatment contingencies were arranged to decrease the rate of stereotypy and increased functional play skills. Overall, our results support the use of latency to respond as a dependent measure for selecting reinforcers.

Keywords: Response Latency, Preference Assessment, Automatic Behavior

When working with children who engage in inappropriate behavior, it is relatively easy to identify a social function. Through observation and manipulation of environmental variables, previous research suggests that most problem behavior is maintained by a social function (Iwata et al., 1994). Treatment for behaviors that serve an automatic function are more difficult to identify because social reinforcers are not identified. Specifically, maintaining contingencies are extremely difficult to manipulate because the behavior itself provides reinforcement (Iwata et al., 1994). Often, an attempt is made to identify activities or objects that can compete with the behavior itself (Alberto \& Troutman, 2005). Alternatively, attempts can be made to identify substitutable stimuli to replace sensory reinforcement obtained by engaging in the behavior (Piazza, Hanley, \& Fisher, 1996). In addition, it has been demonstrated in the literature that substitutable stimuli are (a) preferred and (b) result in decreased levels of problem behavior when presented non-contingently.

The purpose of this study was to identify effective reinforcers for use in a treatment package for a young child who engaged in stereotypic behaviors maintained by automatic reinforcement. By doing so, we further sought to compare the use of choice versus response latency as a dependent measure for use within a preference assessment. We hypothesized that more effective reinforcers would be identified using response latency as a dependent measure. Specifically, because these stimuli were shown to decrease the occurrence of automatic behavior within the preference assessment, we hypothesized that they were substitutable to the behavior.

Method

\section{Participants and Setting}

The participant was a 6-year-old female diagnosed with Rett Syndrome. She was referred by her classroom teacher for the assessment and treatment of stereotypy. Stereotypy was reported to occur at high rates in home and school settings. All observations took place in a 3.m by $4.5 \mathrm{~m}$ room located adjacent to her classroom. The room was equipped with a two-way window for unobtrusive observation.

\section{Data Collection, and Interobserver Agreement}

The dependent variables were (a) inappropriate vocalizations, which were defined as loud, raspy, noises in the back of the throat occurring in brief bursts; (b) spitting which was defined as having the tongue completely out of the mouth making a spitting noise; (c) hand 
wringing which was defined as rubbing the pads on the fingers of one hand against the pads on the fingers of the other hand together, usually done by the shoulder; (d) hand flapping defined as moving her hands up and down either by the hips or shoulders; (e) latency, which was defined as the amount of time, in seconds, between the presentation of an item and the first occurrence of a stereotypic response; and (f) choice, which was defined as reaching for one of two objects within a concurrent operant format.

Data were collected using three different observational systems: (a) partial-interval recording, (b) event recording, and (c) latency recording. All partial interval recording was collected via videotape and scored later using a 6-s recording system. For event recording, live data were taken to measure choice behavior and responses were marked each time a choice occurred. Latency was measured by watching a videotape and recording the number of seconds that had lapsed between presentation of an item and problem behavior. Interobserver agreement was assessed for $56 \%$ of the sessions. An agreement occurred when both observers recorded that a aberrant target behavior occurred in the same interval or that a specific choice was made. Occurrence agreement was computed by dividing agreements by agreements plus disagreements and multiplying by $100 \%$. Average agreement was $88.8 \%$ across all dependent measures.

\section{Experimental Design and Phases}

The investigation was conducted across 3 phases: (a) functional analysis, (b) preference and reinforcer assessment, and (c) treatment of comparison. For Phase 1, a functional analysis (Iwata et al., 1994) was conducted using a multi-element design to determine behavioral function. During Phase 2, a forced-choice preference assessment was conducted in which the participant was required to choose between two preferred items (Fisher et al., 1992). Those items were then used in the reinforcer assessment that was conducted using the procedures described by Derby et al. (1995), in which the latency to the first occurrence of a target behavior was measured. In Phase 3, a combination of differential reinforcement of alternate behavior (DRA) and differential reinforcement of other behavior (DRO) using a resetting time schedule of 30s. This was done to decrease the automatic behaviors of spitting and inappropriate vocalizations. Treatment was conducted within an $\mathrm{ABACBC}$ single case design across three conditions (a) the alone condition from the functional analysis, (b) DRA/O with a resetting time schedule of 30 seconds using a reinforcer identified using the latency measure, and (c) the same DRA/O with resetting time of 30 s using the preferred item identified via the choice measure.

\section{Procedure}

During Phase 1, a functional analysis (Iwata et al., 1994), using free play, attention, alone, escape, and tangible conditions, was conducted to evaluate the effects of the presence and absence of task demands, social attention, and preferred tangibles on the participant's behavior using the procedure described by Iwata et al. (1994). During Phase 2, a forced-choice preference assessment was conducted using the procedures described by Fisher et al. (1992). The items were presented until each pairing had been exhausted and the results identified of an item preference hierarchy. Using this hierarchy, the high, medium, and low preferred items were then evaluated with the reinforcer assessment. The items were again paired, but were alternately presented to the participant. One session consisted of ten presentations (five presentations of each item). Latency between the presentation of the item and the first occurrence of an aberrant behavior was measured in seconds. At the first occurrence of a target response, the item was removed, time was recorded, and the other item in the pairing was presented. The items were alternately presented 
for a 5-min session and access to an item was provided when either a target response occurred or 5-min had elapsed.

The third phase of the study was treatment. An appropriate play skill of rolling a ball was differentially reinforced using one of two items identified in the preference assessment. The therapist sat on the floor behind the participant and physically assisted her in rolling the ball and catching the ball (i.e., helped her put her hand on the ball without interfering with engagement in the aberrant behavior). It should be noted that no attempts were made to redirect her hand away from her mouth when she was assisted with ball rolling or catching. A timer was set for 30s when the ball was continuously rolled. If the participant rolled the ball appropriately without engaging in a target response for 30s, she was presented with either a book or donut, depending on the experimental condition. However, if she engaged in a target response at any time during the 30s interval, the timer was reset for another 30 seconds, and ball rolling continued, without item delivery.

\section{Results and Discussion}

The results of the functional analysis are shown in Figure 1. High rates of behavior were observed across all conditions, with the highest levels occurring in the alone condition, suggesting that problem behavior was maintained by automatic reinforcement. The results of the preference assessment are shown in Figure 2. The 11 preferred items are shown hierarchically. A book was the participant's most preferred item, indicated by the number of times chosen. Small pieces of chocolate donuts were identified as medium preferred item. Music was identified as least preferred item.

The results of the reinforcer assessment are shown in Figure 3. After measuring the latency to the first occurrence of a target behavior, the participant's medium preferred item, donut, was shown to result in the longest response latency, with an average latency of 66 seconds. A book, which was identified as the most preferred item using choice, had a much shorter latency, with an average latency of 36 seconds. And the low preferred item had the shortest average latency with 33 seconds.

The results of treatment are shown in Figure 4. In Phase 1, the alone condition from the functional analysis, very high rates of behavior were observed $(M=83.7 \%$; range $79 \%$ to $88 \%)$. The implementation of DRA/O using the donut as a reinforcer dramatically decreased problem behavior to very low levels $(M=10.2 \%$; range $2 \%$ to $24 \%)$. A reversal back to the alone condition resulted in a sharp increase in problem behavior $(M=88 \%$, range $=80 \%$ to $96 \%)$. Next, the use of the book as a reinforcer resulted in a moderate decrease in target behavior $(M$ $=37.3 \%$; range $=24 \%$ to $46 \%$ ). When the donut was reintroduced as a reinforcer aberrant behavior was dramatically reduced. A reversal back to the book as a reinforcer resulted in problem behavior occurring at a higher rate $(M=19.43 \%$; range $2 \%$ to $30 \%)$.

Overall, our results support the use of latency as a dependent measure. Thus, latency to response may be a useful measure for behaviors that serve an automatic function. That is, if the presentation of the item results in increased response latency it could be hypothesized that it is providing a competing form of sensory stimulation.

In this case, many of the participant's behaviors were oral in nature. Thus, the presentation of the donut probably provided an alternative form of oral sensation. It should be noted that the donut was not simply an incompatible response for problem behavior. Specifically, she could have continued to engage problem behavior with the donut in her mouth. One limitation that should be noted is that we were unable to return to the donut condition at the end 
of the analysis because the school year ended. A second limitation is that we compared a stimulus that was potentially a substitutable oral stimuli (the donut) with a none substitutable stimuli (i.e., the book). Additional research using response latency with potential substitutable and non-substitutable stimuli is needed. Nevertheless, our results indicate that response latency was an effective dependent measure and that additional research is needed.

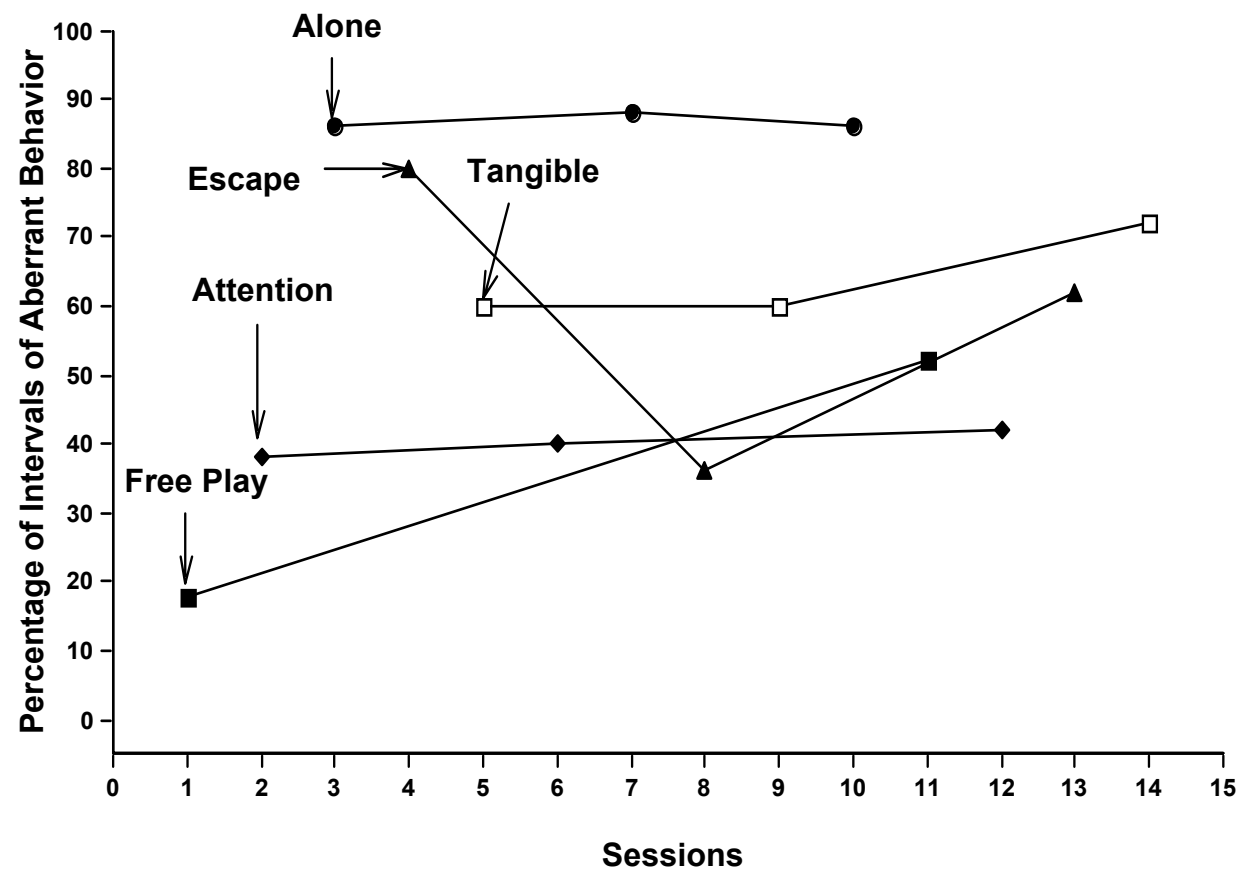

Figure 1. Initial functional analysis results




Figure 2. Choice preference assessment results for the participant. The ** indicates that theses items were reassessed.

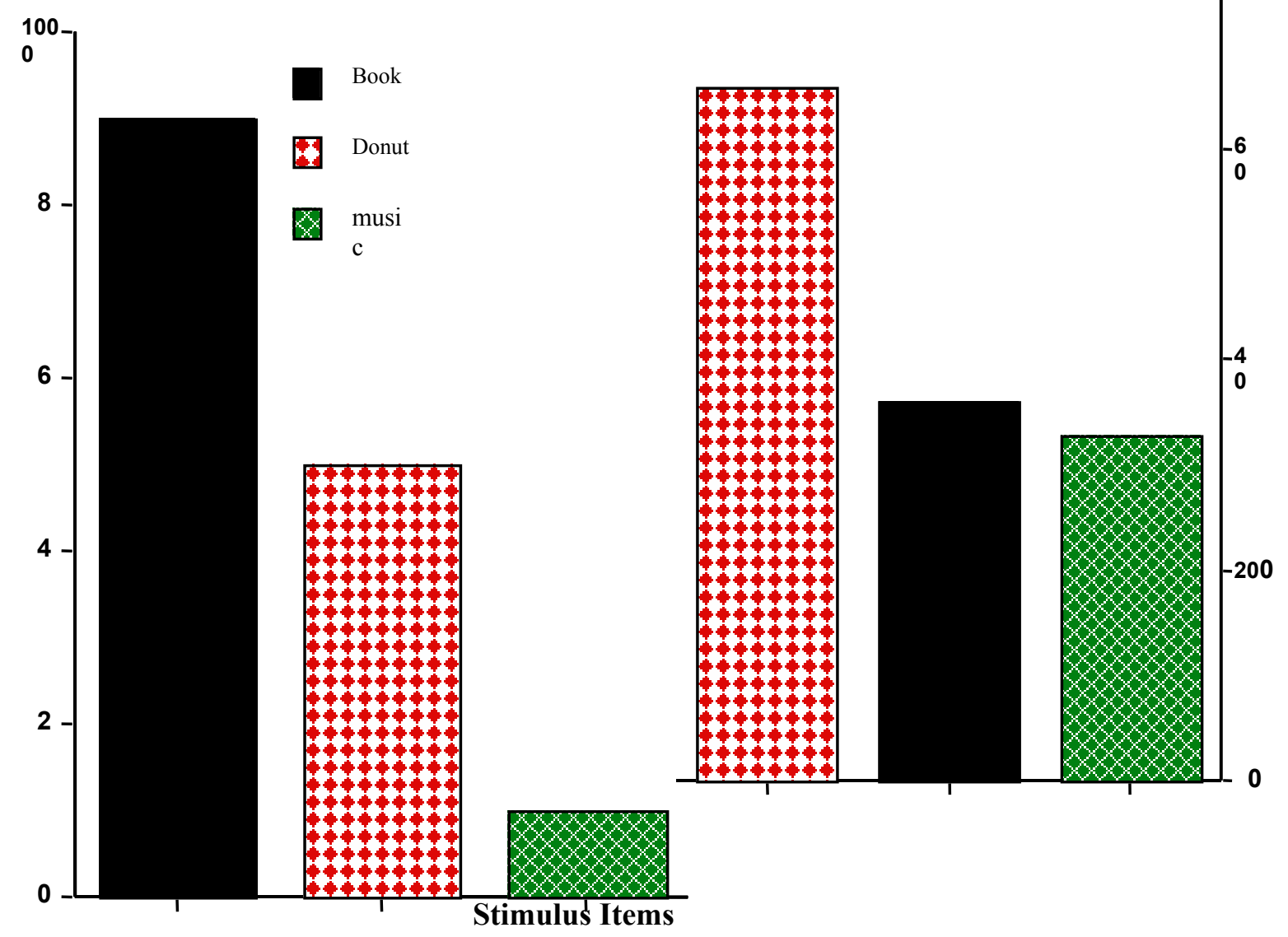

Figure 3. Comparison results obtained for choice (right side) and latency (left side) for aberrant behavior 


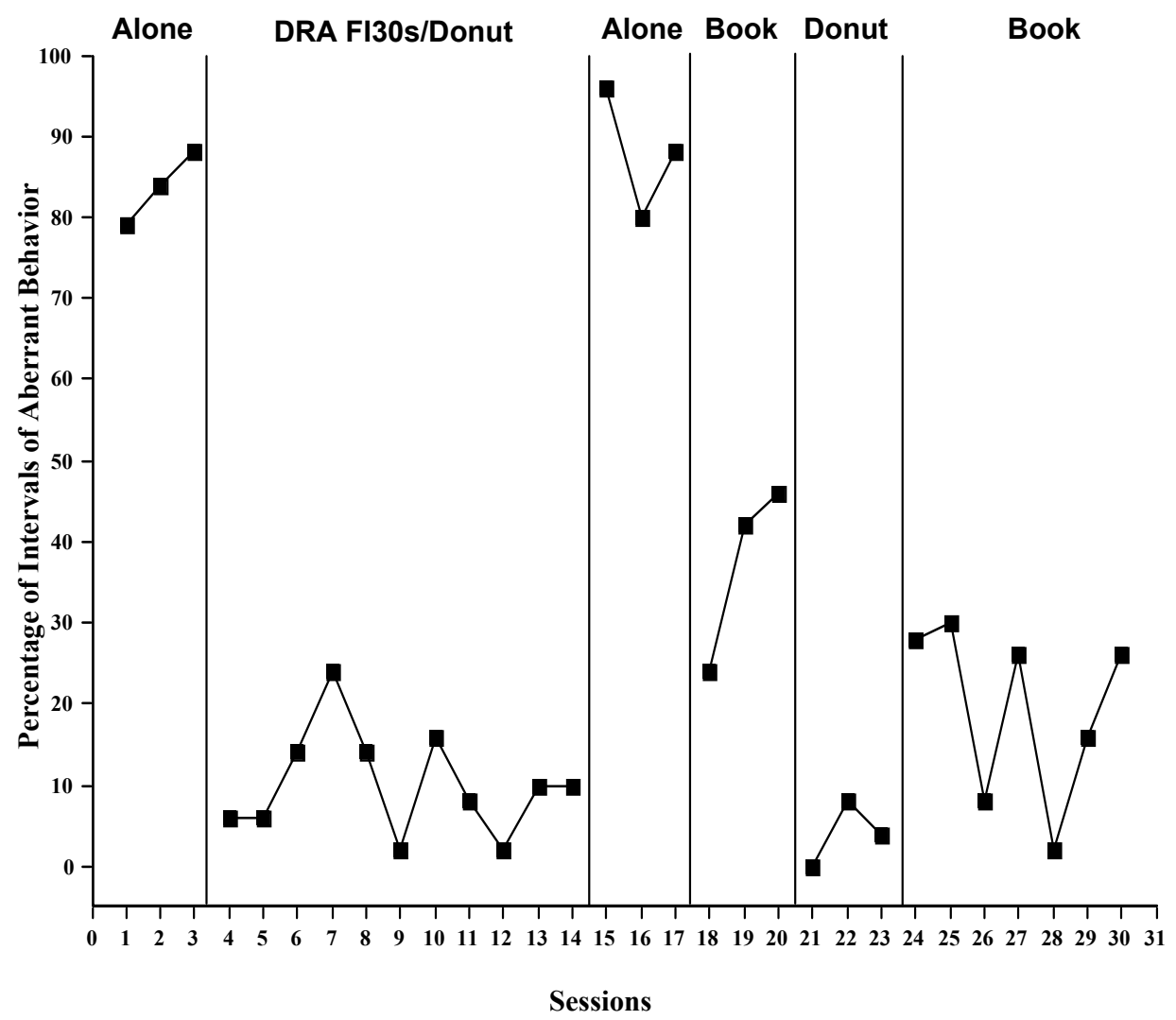

Figure 4. Treatment outcomes.

\section{References}

Alberto, P., \& Troutman, A. C. (2005). Applied behavior analysis for teachers $\left(6^{\text {th }} \mathrm{ed}\right)$. Upper Saddle River NJ: Merrill/Prentice-Hall/Pearson.

Derby, K. M., Wacker, D. P., Andelman, M., Berg, W., Drew, J., Asmus, et al. (1995). Two measures of preference during forced-choice assessments. Journal of Applied Behavior Analysis, 28, 345-346.

Fisher, W., Piazza, C. C., Bowman, L. G., Hagopian, L. P., Owens, J. C., \& Slevin, I. (1992). A comparison of two approaches for identifying reinforcers for persons with severe and profound disabilities. Journal of Applied Behavior Analysis, 25, 491-498.

Iwata, B. A., Pace, G. M., Dorsey, M. F., Zarcone, J. R., Vollmer, T. R., 
Smith, R. G., et al. (1994). The functions of self-injurious behavior: An experimentalepidemiological analysis. Journal of Applied Behavior Analysis, 27, 215-240.

Piazza, C. C., Hanley, G. P., \& Fisher, W. W. (1996). Functional analysis and treatment of cigarette pica. Journal of Applied Behavior Analysis, 29, 437-449

Author Notes:

This investigation was completed in partial fulfillment for the Master of Education in Special Education in Special Education at Gonzaga University by the first author. Requests for reprints should be sent to K. Mark Derby, Professor, Department of Special Education, School of Education, Gonzaga University, Spokane, WA, 99258-0025 or via email at derby@gonzaga.edu

Stephanie K. Meador, Clinical Director at Alpine Autism Center

Colorado Springs

5965 Vista Ridge Pt. \#202

Colorado Springs, CO 80918

719-510-0837

e-mail skmeador@yahoo.com

K. Mark Derby,

Professor

Department of Special Education

Gonzaga University

Spokane WA 99258

509-328-4220

e-mail derby@gonzaga.edu

T. F. McLaughlin,

Professor

Department of Special Education

Gonzaga University

Spokane WA 99258

509-328-4220

e-mail McLaughlin@gonzaga.edu

Anjali Barretto

Associate, Professor

Department of Special Education

Gonzaga University

Spokane WA 99258

509-328-4220

e-mail barretto@gonzaga.edu

Kim Weber

Associate, Professor

Department of Special Education

Gonzaga University

Spokane WA 99258

509-328-4220

e-mail kweber@gonzaga.edu 